\title{
RNA:RNA interaction can enhance RNA localization in Drosophila oocytes
}

\author{
EVE HARTSWOOD, ${ }^{1,3}$ JIM BRODIE, ${ }^{1,3}$ GEORGIA VENDRA, ${ }^{1,4}$ ILAN DAVIS, $^{2}$ and DAVID J. FINNEGAN ${ }^{1,5}$ \\ ${ }^{1}$ Institute of Cell Biology, School of Biological Sciences, University of Edinburgh, Edinburgh EH9 3JR, United Kingdom \\ ${ }^{2}$ Department of Biochemistry, University of Oxford, South Parks Road, Oxford OX1 3QU, United Kingdom
}

\begin{abstract}
RNA localization is a key mechanism for targeting proteins to particular subcellular domains. Sequences necessary and sufficient for localization have been identified, but little is known about factors that affect its kinetics. Transcripts of gurken and the I factor, a non-LTR retrotransposon, colocalize at the nucleus in the dorso-antero corner of the Drosophila oocyte directed by localization signals, the GLS and ILS. I factor RNA localizes faster than gurken after injection into oocytes, due to a difference in the intrinsic localization ability of the GLS and ILS. The kinetics of localization of RNA containing the ILS are enhanced by the presence of a stem-loop, the A loop. This acts as an RNA:RNA interaction element in vivo and in vitro, and stimulates localization of RNA containing other localization signals. RNA:RNA interaction may be a general mechanism for modulating RNA localization and could allow an mRNA that lacks a localization signal to hitchhike on another RNA that has one.
\end{abstract}

Keywords: Drosophila oocyte; I factor; RNA localization; RNA:RNA interaction; gurken

\section{INTRODUCTION}

RNA localization is a widespread and important mechanism by which cells confine functions to particular subcellular domains. Active transport of RNA along cytoskeletal networks, often of microtubules (MTs), leads to spatially restricted protein expression with the destination of particular RNAs often being determined by localization signals that are recognized by proteins required for transport (Becalska and Gavis 2009). RNAs localized in this way are incorporated into transport particles together with cargo-binding proteins and motor proteins that move the particles along the cytoskeleton (Fusco et al. 2003; Bullock and Bedau 2006; Bullock 2007; Delanoue et al. 2007; Becalska and Gavis 2009). Recognition of localization motifs within an RNA, incorporation of RNA and associated proteins into transport particles, and movement of the particles along the cytoskeleton, are each potential targets for the control of RNA localization.

Localization of RNA coding for the TGF- $\alpha$-related protein Gurken (Grk) is required to establish both the anterior-posterior

\footnotetext{
${ }^{3}$ These authors contributed equally to this work.

${ }^{4}$ Present address: Centre for Brain Research, Medical University of Vienna, Spitalgasse 23, A-1090 Vienna, Austria.

${ }^{5}$ Corresponding author.

E-mail david.finnegan@ed.ac.uk.

Article published online ahead of print. Article and publication date are at http://www.rnajournal.org/cgi/doi/10.1261/rna.026674.111.
}

and dorsal-ventral axes of Drosophila oocytes and embryos (Neuman-Silberberg and Schupbach 1993; Tomancak et al. 1998). grk RNA is transcribed in nurse cells from where it is transported to the oocyte (Clark et al. 2007). At stage 6-7 of oogenesis (Spradling 1993) it is concentrated at one end of the oocyte, where it is translated to establish posterior fate. It is then moved to the anterior of the oocyte, and from there to the dorso-anterior corner where, at stage 9, it forms a cap over the nucleus. In each case, grk RNA is moved to the minus end of microtubules by cytoplasmic dynein (MacDougall et al. 2003). Once at its final destination it is anchored by a mechanism requiring both dynein and squid (Delanoue et al. 2007), and is again translated, this time specifying the dorsal side of the oocyte (Riechmann and Ephrussi 2001). The movement of endogenous grk RNA can be recapitulated by injecting fluorescently labeled RNA into oocytes (Fig. 1A; MacDougall et al. 2003; Van De Bor et al. 2005). This has allowed identification of a sequence, the grk localization signal (GLS), which is both necessary and sufficient for localization of grk RNA (Van De Bor et al. 2005). The GLS is predicted to form a stem-loop (Fig. 1B) and is recognized in vitro by the cargo-binding protein Egalitarian (Dienstbier et al. 2009), which together with BicD, is required for grk localization (Navarro et al. 2004). Although the GLS is sufficient to direct the dorso-anterior localization of RNA injected into the oocyte, Lan et al. (2010) have suggested that this may not be the case for RNA expressed endogenously from the grk promoter. 
The putative RNA transposition intermediate of the I factor, a non-LTR retrotransposon of Drosophila (Fawcett et al. 1986; Bucheton et al. 2002), is also transcribed in nurse cells and localized in the oocyte with a pattern indistinguishable from that of grk (Fig. 1C; Van de Bor and Davis 2004; Seleme et al. 2005). We have identified a sequence, the I factor localization signal (ILS), that is both necessary and sufficient for localization of I factor RNA in oocytes (Fig. 1D). This is predicted to form a stem-loop similar in structure (Fig. 1D), but not in sequence, to that of the GLS (Van De Bor et al. 2005; Hamilton et al. 2009), suggesting that grk and I factor RNAs may use overlapping sets of transport proteins for localization. This is supported by our observation that injection of an excess of RNA containing one of these localization signals interferes with the localization of RNA containing the other (Van De Bor et al. 2005). Despite the similarity between the GLS and ILS, grk and I factor RNAs did not move to the dorso-anterior corner of the oocyte with the same kinetics after injection into stage 9 oocytes, as RNA containing the ILS took less time to localize than did RNA containing the GLS (Van De Bor et al. 2005). We have now found that this difference can be attributed to the kinetics of localization associated with the ILS and GLS themselves, and that this can be modulated by other features of the RNA containing them. In the case of $I$ factor RNA, this is a second stem-loop, the A loop (Fig. 1D), which enhances localization mediated by the ILS. This effect is not confined to the ILS, as it is seen with RNAs carrying other localization signals. We propose that this reflects a general mechanism by which the intracellular behavior of RNA is modulated through self-association sequences that pro-
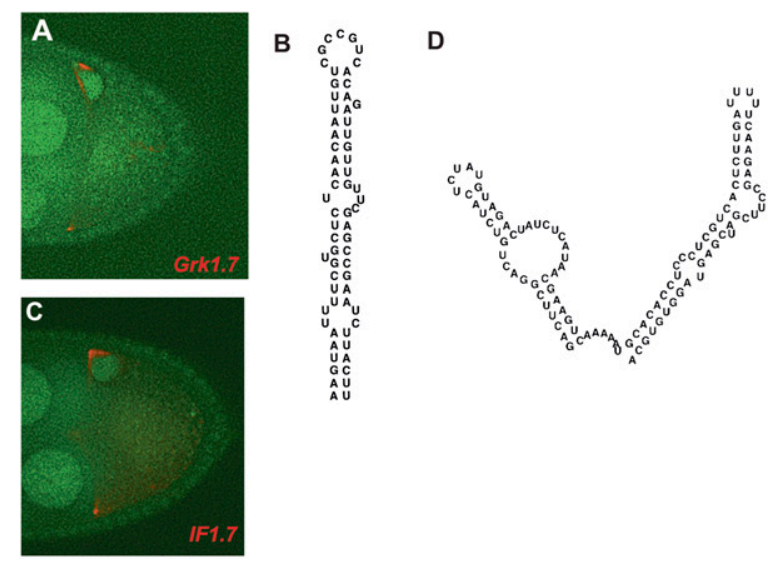

FIGURE 1. Localization of Grk1.7 and IF1.7 RNA. (A) Localization of Grk1.7 RNA (red) after injection into the center of a stage 9 oocyte from a female expressing nls-GFP. Anterior is to the left and dorsal to the top. The RNA can be seen forming a cap over the oocyte nucleus, marked with nls-GFP, at the dorso-anterior position of the oocyte. Two nurse cell nuclei can be seen anterior to the oocyte. (B) The sequence GLS shown with the secondary structure predicted by RNALFold (Hofacker et al. 2004). (C) Localization of IF1.7 RNA (red). (D) The sequence of the A loop and of the ILS shown with the secondary structure predicted by RNALFold. mote the formation of large RNP particles. Such sequences would also allow mRNAs that lack localization signals to hitchhike on localizing transcripts.

\section{RESULTS AND DISCUSSION}

\section{I factor RNA localizes more efficiently than grk RNA}

The GLS and ILS were identified by live imaging of fluorescently labeled RNA injected into oocytes. The results of these experiments suggested that I factor RNA localized to the dorso-anterior corner of the oocyte faster than did grk RNA (Van De Bor et al. 2005). We have repeated these experiments and have found that the time taken to reach full localization, the interval between injection of RNA at the center of an oocyte to the time beyond which no further change in the localization pattern could be detected, is significantly less $\left(P=10^{-8}\right)$ for IF1.7, a $1.7-\mathrm{kb}$ RNA containing the ILS, than for Grk1.7, a 1.7-kb grk RNA containing the GLS (Fig. 2C,D). The structure of Grk1.7, IF1.7, and of each of the other RNAs used in these experiments, is indicated in Supplemental Figure S1.

There are several factors that, singly or in combination, could account for the difference in the kinetics with which grk and I factor RNAs localize. These include the efficiency with which the RNA is assembled into transport particles, the average distance traveled by a particle along a microtubule in a single run (the run length), the speed with which each particle moves, and the path taken by each particle to get to its destination. The paths taken by grk and I factor transcripts to arrive at the oocyte nucleus are similar, with both moving first to the anterior face of the oocyte and then dorsally to the nucleus (MacDougall et al. 2003; Van De Bor et al. 2005). The average run length of particles containing IF1.7 RNA was greater than that of particles containing GLS1.7 RNA (Fig. 2A), although this difference was not significant at the $95 \%$ confidence level $(P=0.087)$. In contrast, IF1.7 containing particles moved 1.3 times faster than Grk1.7 particles (Fig. $2 \mathrm{~B}, P=6.7 \times 10^{-8}$ ), indicating that this is an important factor contributing to the difference in their time of localization.

As oocytes of Oregon $\mathrm{R}$ females, the strain used for these experiments, express endogenous grk RNA but not full-length I factor RNA, it was possible that transport of injected grk RNA might have been reduced because it had to compete with endogenous transcripts for one or more transport factors. We can exclude this possibility, as particles containing Grk1.7 RNA did not move any faster $(0.53 \pm 0.02 \mu \mathrm{m} / \mathrm{sec})$ in oocytes of $g r k^{E 12} / g r k^{2 B 6}$ flies that do not express $g r k$ RNA than they did in $g r k^{+}$oocytes $(0.55 \pm 0.19 \mu \mathrm{m} / \mathrm{sec})$. IF1.7 RNA also moved at the same speed $(0.77 \pm 0.02 \mu \mathrm{m} / \mathrm{sec})$ in both.

We conclude that IF1.7 RNA takes less time to localize to the dorso-anterior corner of the oocyte than does GRK1.7 RNA, primarily because particles containing IF1.7 RNA move along MTs more rapidly. 
A

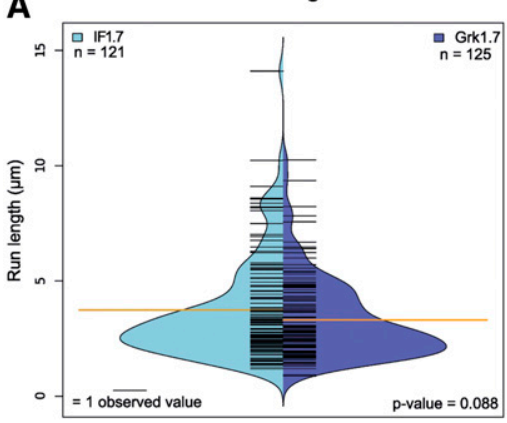

B

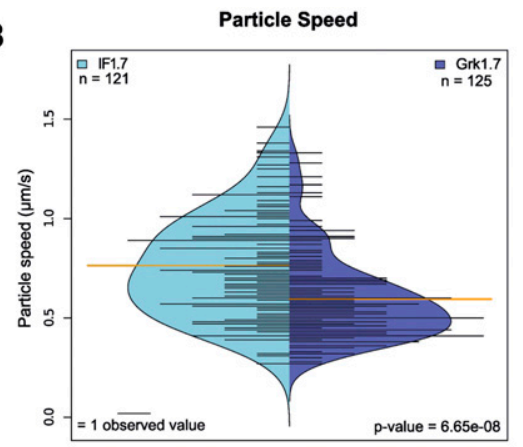

C

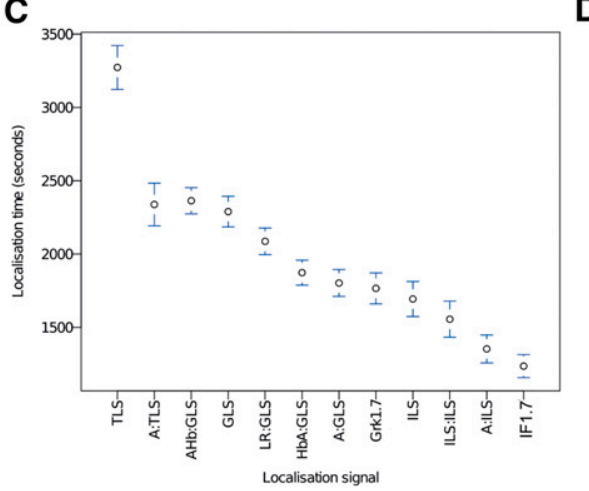

D

\begin{tabular}{|c|c|}
\hline $\begin{array}{c}\text { Localising } \\
\text { RNA }\end{array}$ & $\begin{array}{c}\text { Mean Localisation } \\
\text { Time } \pm \text { S.E.M. sec. }\end{array}$ \\
\hline TLS & $3273 \pm 73(n=30)$ \\
\hline A:TLS & $2338 \pm 72(n=34)$ \\
\hline A:Hb:GLS & $2363 \pm 44(n=33)$ \\
\hline GLS & $2289 \pm 51(n=35)$ \\
\hline LR:GLS & $2087 \pm 44(n=31)$ \\
\hline Hb:A:GLS & $1873 \pm 42(n=31)$ \\
\hline$A: G L S$ & $1802 \pm 45(n=30)$ \\
\hline Grk1.7 & $1766 \pm 51(n=28)$ \\
\hline ILS & $1693 \pm 59(n=31)$ \\
\hline ILS:ILS & $1555 \pm 60(n=30)$ \\
\hline$A: I L S$ & $1353 \pm 46(n=29)$ \\
\hline IF1.7 & $1236 \pm 38(n=31)$ \\
\hline
\end{tabular}

FIGURE 2. Speed and localization time of RNA injected into oocytes. Particle run length $(A)$ and particle speed (B) for Grk1.7 and IF1.7 RNA injected into stage 9 oocytes. The distribution of measurements for individual particles are shown using a bean plot (Kampstra 2008). The horizontal lines indicate values for individual particles, and the distribution of values is indicated by the dark- (Grk1.7) or light- (IF1.7) blue surface. (Orange bar) Mean value for each distribution. The average run length was $3.71 \pm 0.20 \mu \mathrm{m}$ for particles containing IF1.7 RNA, and $3.31 \pm 0.15 \mu \mathrm{m}$ for particles containing Grk1.7 RNA. The average particle speed was $0.77 \pm 0.023 \mu \mathrm{m} / \mathrm{sec}$ for IF1.7 RNA, and $0.58 \pm 0.019 \mu \mathrm{m} / \mathrm{sec}$ for Grk1.7 RNA. (C) The distribution of the mean values ( \pm SEM) of the time (in seconds) between injection of an RNA and the formation of a stable distribution at the dorso-anterior corner of the oocyte. The structures of these RNAs are shown in Supplemental Figure S1 and are described in the text. The features that they contain are as follows: (TLS) Localization signal from k10; (A) A loop; ( $\mathrm{Hb}) 300 \mathrm{nt}$ of hunchback sequence; (GLS) localization signal from grk; (LR) dimerization motif from a Group I intron; (Grk1.7) 1.7 nt of grk cDNA sequence containing the GLS; (ILS) localization signal from I factor; (I1.7) $1.7 \mathrm{nt}$ of I factor sequence including the ILS. The value of the mean localization time $( \pm \mathrm{SEM})$ for each RNA is shown in $D$.

\section{A stem-loop enhances RNA localization}

The difference between the localization times of grk and I factor RNAs is not attributable solely to the properties of the ILS and GLS, as RNA containing the ILS but no other I factor sequence (ILS RNA) localized more slowly (Fig. 2C,D) than did IF1.7 RNA $\left(P<10^{-7}\right)$. The region of $I$ factor RNA containing the ILS is predicted to be rich in stable hairpins (Seleme et al. 2005). None of these localize in oocytes in the absence of the ILS (Van De Bor et al. 2005), but one of them, the A loop (Fig. 1D), stimulates transport. The time taken for RNA containing both the ILS and the A loop (A:ILS RNA) to localize after injection was similar $(P=$ $0.84)$ to that of IF1.7 RNA, but 0.8 times $\left(P=1.5 \times 10^{-4}\right)$ that of ILS RNA (Fig. 2C,D). The effect of the A loop on localization time is not confined to RNA containing the ILS as A:GLS RNA, in which we have introduced the A loop upstream of the GLS, localized in $0.79\left(P=3.5 \times 10^{-5}\right)$ the time taken by RNA containing the GLS alone (Fig. 2C,D). The A loop had a similar effect on RNA containing the TLS, the motif that has a predicted secondary structure similar to those of the GLS and ILS, which directs localization of K10 RNA to the anterior of the oocyte (Cohen et al. 2005). A:TLS RNA localized in 0.71 $\left(P=<10^{-7}\right)$ the time taken by RNA containing the TLS alone (Fig. 2C,D).

Although A:GLS RNA localized more rapidly than RNA with the GLS alone, it still took $1.33\left(P=1.5 \times 10^{-4}\right)$ times as long as A:ILS RNA. There was a similar difference (1.35-fold, $P=2 \times 10^{-7}$ ) between the localization times of RNAs containing either the GLS or ILS alone (Fig. 2C,D). This may reflect a difference in the affinity of transport proteins for the GLS and the ILS. Indeed, recombinant Egalitarian and BicD (Mach and Lehmann 1997; Bullock and Ish-Horowicz 2001) bind more readily to the ILS than to the GLS (Dienstbier et al. 2009). Other transport proteins may well be involved, as TLS RNA took much longer to localize than GLS RNA, even though it is bound more strongly by Egalitarian and BicD protein in vitro (Dienstbier et al. 2009).

Proximity of the A loop to a localizing signal appears to be critical, as $A: H b: G$ RNA in which the A loop is separated from the GLS by $300 \mathrm{nt}$ of Hunchback, an RNA that does not itself localize within oocytes (Davis and Ish-Horowicz 1991; Wilkie et al. 2001), localized with similar kinetics $(P=0.99)$ as RNA containing the GLS alone (Fig. 2C,D). This is not an effect of the Hunchback sequence per se as Hb:A:GLS RNA, with the $\mathrm{Hb}$ sequence upstream of the A loop, took a similar time $(P=0.99)$ to localize as did A:GLS RNA (Fig. $2 \mathrm{C}, \mathrm{D})$.

\section{The A loop mediates RNA:RNA interaction in vivo and in vitro}

The A loop could enhance RNA localization by interacting with one or more factors that stimulate transport, or by allowing interaction between RNAs that contain a localization signal, thereby facilitating their recognition by transport proteins, or both. 
Intermolecular interaction appears to affect the localization of several RNAs in Drosophila embryos (Ferrandon et al. 1997; Bullock et al. 2003; Najand and Simmonds 2007) or oocytes (Hachet and Ephrussi 2004), and we have investigated the possibility that the A loop mediates a similar RNA:RNA interaction. To do this we coinjected I552 $I L S$ RNA, containing the A loop, but with the ILS deleted, together with a second RNA either containing both the ILS and the A loop (A:ILS RNA), or with two copies of the ILS but no A loop (ILS:ILS RNA). The I552 $I L S$ RNA was labeled with Alexa 546 (red), while A:ILS and ILS:ILS RNAs were labeled with Alexa 488 (green). The I552 $I L S$ RNA moved to the nucleus in oocytes when coinjected with A:ILS RNA (Fig. 3A'), but not if injected on its own (0/45 injections) nor when coinjected with ILS:ILS RNA, even though the ILS:ILS RNA moved to the nucleus as expected (Fig. 3B $\mathrm{B}^{\prime \prime}$ ). Colocalization of I552 $\Delta I L S$ and A:ILS RNA appears to be due to interaction between them as they are incorporated into the same transport particles (Fig. 3C; Supplemental data, movie 1). This interaction requires the presence of an A loop on both RNAs, as I552 $I L S$ RNA is not incorporated into transport particles when injected with ILS:ILS RNA (Fig. 3D; Supplemental data, movie 2).

In order to test whether RNAs containing the A loop can interact directly rather than requiring a bridging RNA or protein, we have investigated the ability of RNAs, either with or without the A loop, to form dimers in vitro. RNA was transcribed in vitro, heated to $95^{\circ} \mathrm{C}$ at a range of concentrations, cooled on ice, and then incubated for 30 $\min$ at $37^{\circ} \mathrm{C}$ before electrophoresis on a nondenaturing $1 \%$ agarose gel. The position and intensity of the bands of RNA were then determined using a LI-COR Odyssey infrared imager after staining with SYTO 60. RNAs containing the A loop gave a slowly migrating band seen after incubation at higher concentrations, suggesting that it is a multimeric form, presumably a dimer. This band was not seen with the corresponding RNA without the A loop (shown for $H b: A$ :GLS and $H b$ :GLS RNAs in Fig. 4A). When $H b$ :A:ILS RNA was incubated at a fixed concentration the putative dimer band increased in intensity with time in a nonlinear manner as expected for a multimeric form (Fig. 4B). The most abundant and most rapidly migrating band in Figure $4 \mathrm{~A}$ is presumably the monomer, while bands 2 and 3, between the putative dimer and monomer, probably correspond to the minor products of in vitro transcription that were seen when the RNAs were run on a denaturing gel (Fig. 4C). A putative dimer band was detected with each RNA containing the A loop, but not with the equivalent RNA without the A loop, indicating that the A loop can mediate intermolecular RNA:RNA interaction directly. Although it is tempting to speculate that the dimerization ability of RNA containing the A loop is responsible for the in vivo interaction of RNAs containing it (Fig. 3), and this, in turn, is responsible for the effect of the A loop on RNA localization, either or both of these could require an
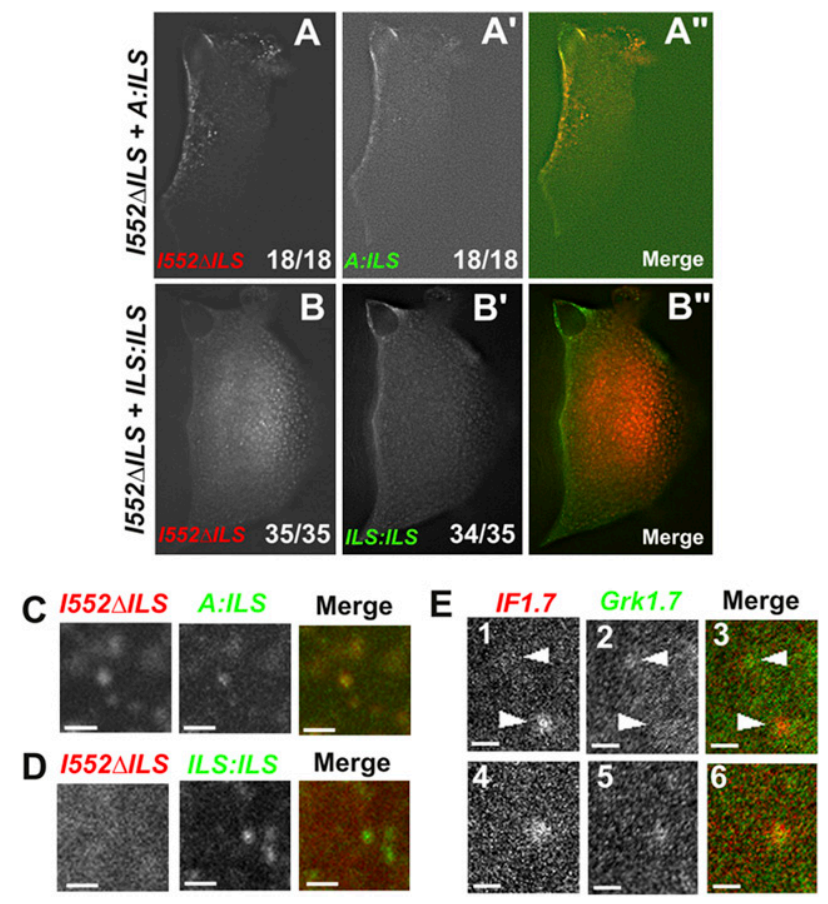

FIGURE 3. RNAs containing the A loop colocalize after injection into the oocyte. The distribution of $(A) I 552 \Delta I L S$ RNA (red) and $\left(A^{\prime}\right)$ A:ILS RNA (green) RNAs after they have been coinjected. $\left(A^{\prime \prime}\right)$ The same oocyte after merging the red and green channels. The distribution of (B) I552 $I L S$ RNA (red) and $\left(B^{\prime}\right)$ ILS:ILS RNA (green) RNAs after they have been coinjected. $\left(B^{\prime \prime}\right)$ The oocyte after merging the red and green channels. The number of injected oocytes giving the pattern shown is indicated at the bottom of $A, A^{\prime}, B$, and $B^{\prime}$. Anterior is to the left and dorsal to the top. In $A^{\prime \prime}$ the ratio of the green versus red signal is 0.40 at the dorso-anterior corner and 0.26 in the center of the oocyte, whereas the green:red ratio in $B^{\prime \prime}$ is 1.2 at the dorso-anterior corner and 0.20 in the center. $(C)$ Transport particles formed after coinjection of I552 $I L S$ RNA (red) and A:ILS RNA (green). (D) Transport particles formed after coinjection of I552 $I L S$ RNA (red) and ILS:ILS RNA (green). (E) Transport particles formed after coinjection of IF1.7 RNA (red) and Grk1.7 RNA (green). (Panels 13) Particles containing either IF1.7 alone (lower particle) or Grk1.7 alone (upper particle). (Panels 4-6) Particle containing both RNAs. The size bar in $1-6$ represents $1 \mu \mathrm{m}$.

additional RNA or protein to recognize the A loop and mediate its effect.

\section{The A loop stimulates formation of transport particles}

Although the ILS is necessary and sufficient for correct positioning of I factor RNA within the oocyte, its action is enhanced by the A loop. Intermolecular RNA interactions may be a common feature of localizing RNAs, perhaps to facilitate their recognition by transport proteins and, hence, their incorporation into transport particles, or to stabilize the particles once assembled. We have compared the size and fluorescence intensity of transport particles formed after injection of either $H b$ :GLS or $H b: A$ :GLS RNA. Those containing $H b$ :A:GLS RNA are both bigger $(46.7 \pm 3.24$ versus $32.0 \pm 1.42$ pixels, $P=1.4 \times 10^{-4}$ ) and brighter 

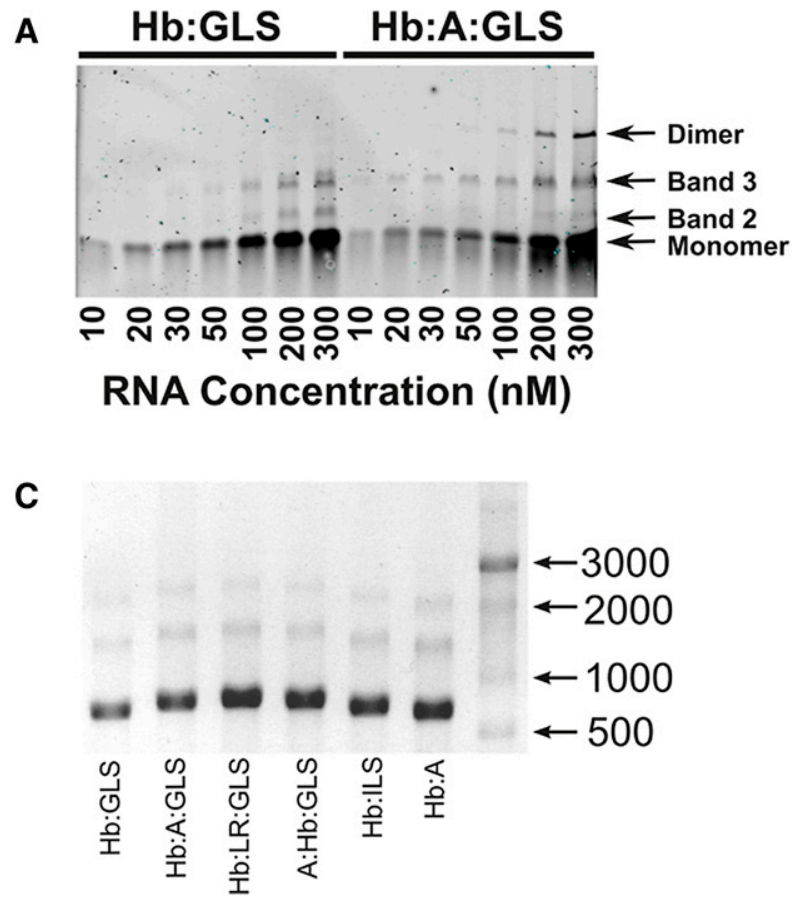

B

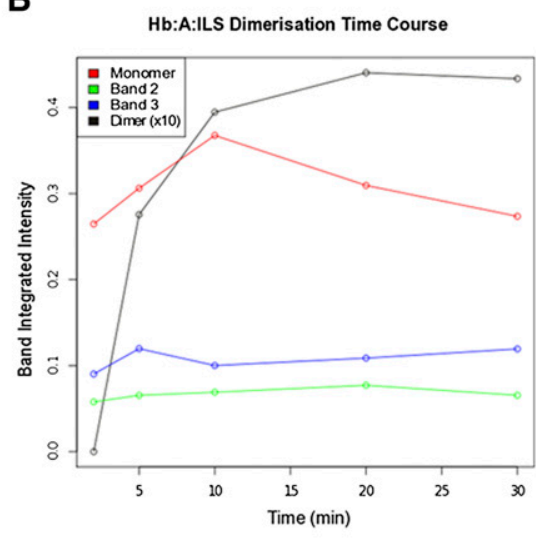

FIGURE 4. In vitro dimerization of RNA containing the A loop. (A) $H b$ :GLS and $H b: A$ :GLS RNA fractionated on a $1 \%$ nondenaturing gel after incubation at $37^{\circ} \mathrm{C}$ for $30 \mathrm{~min}$, using the concentrations indicated. The RNA was stained with SYTO-60 and visualized and quantified with a LICOR Odyssey infrared imager. The arrow indicates the position of the putative dimer band. $(B)$ Products of in vitro transcription fractionated on a denaturing agarose gel. The templates used to generate these RNAs are shown in Supplemental Figure S1. The most intense band corresponds to RNA of the expected size. (C) Hb:A:ILS dimerization in vitro. A total of $200 \mathrm{nM} \mathrm{Hb:A:ILS} \mathrm{RNA} \mathrm{was} \mathrm{heated} \mathrm{to} 95^{\circ} \mathrm{C}$, cooled on ice, and incubated at $37^{\circ} \mathrm{C}$ for the times shown and fractionated on a $1 \%$ nondenaturing agarose gel. The gel was stained with SYTO 60 and the intensity of bands measured using a LI-COR Odyssey infrared imager. The change in the intensity of each molecular form with time is indicated in the graph.

$(1440 \pm 67.8$ versus $1100 \pm 73.1$ grayscale units, $P=1.1 \times$ $10^{-3}$ ) than those formed after injection of $H b$ :GLS RNA (Fig. 5A,B). The correlation between particle size and particle intensity (Fig. 5C) is also stronger $(\mathrm{r}=0.70, P=$ $\left.1.4 \times 10^{-6}\right)$ for $H b: A: G L S$ containing particles than for those with $H b$ :GLS RNA ( $\mathrm{r}=0.13, P=0.40$ ), suggesting that the presence of the A loop results in a more coordinated incorporation of RNA into transport particles. The larger particles formed by $H b: A$ :GLS RNA may contain more copies of dynein, or of one or more transport proteins that stimulate dynein, allowing these particles to move more rapidly along MTs.

The difference in the localization times of IF1.7 and Grk1.7 RNAs coinjected into oocytes (Van De Bor et al. 2005) could reflect assembly of these RNAs into separate transport particles that move at different rates, or the random assembly of the two RNAs into transport particles, but with a bias favoring IF1.7 RNA. In order to distinguish between these possibilities we have analyzed transport particles formed after coinjection of differentially labeled IF1.7 and Grk1.7 RNA. Three populations of particles were visible. Those with either IF1.7 or Grk1.7 (Fig. 3E, 1-3), and those with both (Fig. 3E, 4-6). Although we could not accurately measure the proportion of each class of particle, those containing IF1.7 RNA tended to be larger and brighter than those with Grk1.7 alone. This was the case whether IF1.7 was labeled with Alexa 546 and Grk1.7 with Alexa 488, or the reverse, indicating that both IF1.7 and Grk1.7 RNAs can be assembled into the same transport particle, but that IF1.7 RNA is incorporated into particles more efficiently. This may be due to a difference in the efficiency with which the ILS and GLS interact with transport proteins, including Egalitarian and BicD. Dimerization of IF1.7 via the A loop could accentuate this difference, since two or more RNA molecules could be incorporated into a particle via a single protein:ILS interaction. Transport by particles containing more IF1.7 than Grk1.7 RNA would lead to more rapid accumulation of IF1.7. This suggests that there are least two factors that may contribute to the different kinetics with which IF1.7 and Grk1.7 localize, the efficiency of incorporation into transport particles, and the speed with which particles move. The latter will affect the rate of accumulation of RNAs traveling to their destination in particles containing only one or the other RNA, while the former will affect accumulation of RNA transported by particles containing both.

RNA:RNA interaction may be a common mechanism modulating the effect of RNA localization signals. RNA sequences predicted to form stem loops enhance the apical localization of wingless (Najand and Simmonds 2007) and hairy RNA in Drosophila embryos (Bullock et al. 2003) without themselves being able to direct localization, and in 
A

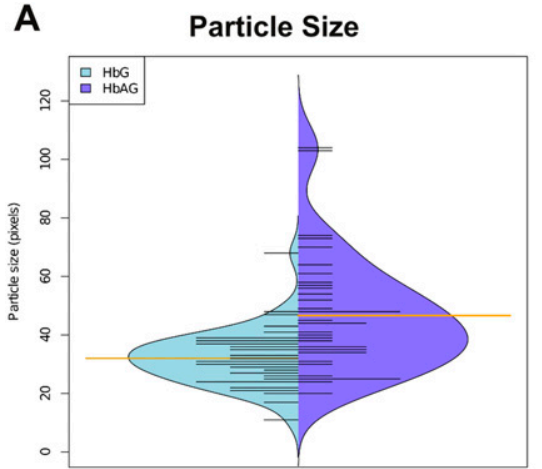

B

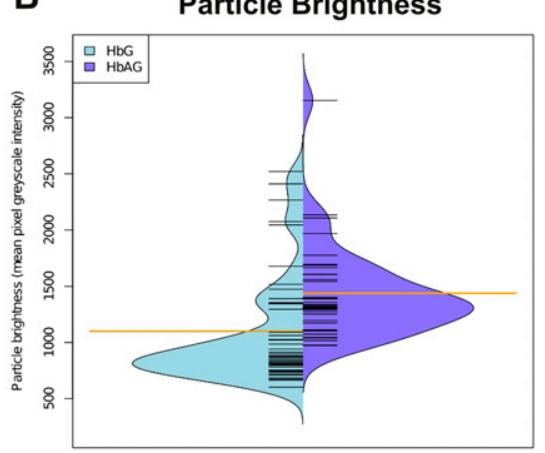

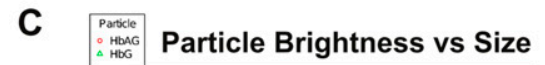

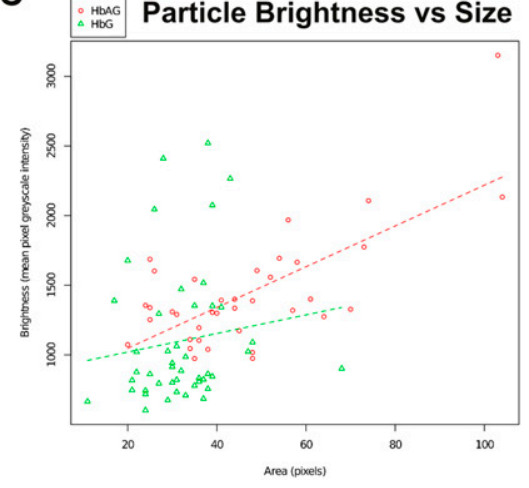

FIGURE 5. The presence of the A loop increases particle size and intensity. Bean plots showing $(A)$ the distribution of the mean number of pixels; $(B)$ the mean grayscale pixel intensity of transport particles containing Hb:GLS RNA (left) or Hb:A:GLS RNA (right). (C) The relationship between particle size and particle intensity for particles containing either $H b$ :GLS RNA (green) or $H b: A: G L S$ RNA (red).

each case this appears to be due to the ability to stimulate RNA:RNA interaction. Localization of bicoid mRNA to the anterior of oocytes and embryos requires binding of the Staufen protein to a region of the bicoid 3' UTR (St Johnston et al. 1989; Ferrandon et al. 1994). This region is predicted to form stem loops, and base-pairing between these loops leads to an intermolecular interaction that allows Staufen binding and transport of the RNA along MTs (Ferrandon et al. 1994, 1997). The initial step does not require a specific RNA sequence, as other interacting RNA motifs, including the loop-receptor (LR) module (Jaeger et al. 2001) based on dimerization motifs from a Group I intron of Tetrahymena thermophila (Cech 1990), can initiate the process if introduced into the bicoid 3' UTR (Wagner et al. 2001). The localization time for LR:GLS RNA was less than that of GLS (Fig. 2C,D), as would be the case if RNA:RNA interaction stimulates localization, although this was not significant at the $95 \%$ confidence level $(P=0.1)$.

Localization of oskar (osk) RNA to the posterior pole of oocytes (Ephrussi et al. 1991; Kim-Ha et al. 1991) is also associated with RNA:RNA interaction. Normal osk localization requires both a cis-acting sequence in the $3^{\prime}$ UTR of oskar mRNA (Rongo et al. 1995) and at least two components of the exon junction complex bound to the mRNA near the site of the first intron (Hachet and Ephrussi at that site.
2001, 2004). RNA with the osk 3' UTR, but not the components of the exonjunction complex, can localize to the posterior pole in the presence of endogenous osk mRNA apparently by hitchhiking with it (Hachet and Ephrussi 2004). This involves an interaction between the osk 3' UTR sequence on both RNAs mediated by an RNA interaction element like the A loop (Jambor et al. 2011) or a protein such as PTB (Besse et al. 2009) that binds to sites on the $3^{\prime}$ UTR.

grk RNA does not contain an RNA:RNA interaction sequence, as Grk1.7DGLS RNA from which the GLS has been deleted did not localize with Grk1.7 RNA when the two were coinjected into oocytes, even though the Grk1.7 RNA localized as expected (Supplemental Fig. S2). Nevertheless, Grk1.7 RNA localizes more rapidly than RNA containing the GLS alone $\left(P=10^{-5}\right)$ (Fig. 2C,D), indicating that some other feature of grk RNA enhances localization driven by the GLS. This is unlikely to be due simply to difference in size between the Grk1.7 and GLS RNA, as there is no significant difference $(P=0.83)$ between the localization times of IF1.7 RNA and A:ILS RNA that differ in size by a similar amount (Fig. 2C,D). A plausible alternative explanation is that one or more transport factors bind to grk RNA outside of the GLS to enhance the kinetics of localization. Indeed, the ratio of the localization times of IF1.7 and Grk1.7 RNAs (0.74) is similar to that of ILS and GLS RNAs (0.70), indicating that whatever this factor is, it has a similar effect to that of the A loop.

The ability of RNAs that share an interaction motif to assemble into transport particles together could explain why it has been difficult to identify localization motifs common to RNAs that localize to the same site. An RNA that would otherwise be unable to localize may hitchhike on transport particles formed by a second RNA that has a localization signal, with which the first RNA can interact. The ability of RNAs to hitchhike in this way could provide a mechanism to ensure that RNAs coding for proteins that act in concert at a particular site within a cell arrive together

\section{MATERIALS AND METHODS}

\section{Nucleic acid sequences}

The sequence organization of each RNA used in these experiments is shown in Supplemental Figure S1. The GLS is nucleotides 399-463 of 
the grk cDNA, GenBank Accession L22531.1. The A loop is nucleotides 3287-3331, and the ILS is nucleotides 3337-3394 of the I factor, EMBL Accession M14954. The hunchback sequence is nucleotides 2106-2413 of the cDNA, GenBank accession BT024234.1. The sequence of the loop receptor module (GGGATATGGAAGGTT TGGGTTCCTTTCCGGGGAAACTTGGTTTGGGAACCTTTCCT TCCTAAGTCCT) is taken from Figure 7A of Wagner et al. (2001).

\section{Plasmid construction and in vitro transcription}

RNA was transcribed in vitro from linearized plasmid DNA and then purified on an agarose gel. IF1.7 RNA was transcribed from the 3.0-kb KpnI-ClaI fragment of the I factor cloned in pBluescriptSK, a gift of J. Soetaert, after being cut with BsmI. Grk1.7 RNA was transcribed from grk cDNA cloned in pBluescriptIIKS (NeumanSilberberg and Schupbach 1993) cut with NotI. For the remaining RNAs, plasmids were constructed by cloning PCR fragments containing a localization signal into linear pGEM-T Easy (Promega, http://www.promega.com/vectors/pgemtez.txt). The A loop or the Hunchback $(\mathrm{Hb})$ sequence was cloned into the resulting plasmids as necessary. The structure of each construct was confirmed by DNA sequencing. These plasmids were cut with PvuII before RNA synthesis.

In vitro transcription was carried out by a modification of the method of Wilkie and Davis (Wilkie et al. 2001). RNA was fluorescently labeled using UTP conjugated to Alexa 488, Alexa 546 (Invitrogen), or Cy3 (Perkin Elmer). RNA used for localization experiments was synthesized in reactions containing $40 \mu \mathrm{M}$ modified UTP and $400 \mu \mathrm{M}$ UTP. For particle tracking experiments, RNA was synthesized in reactions with $50 \mu \mathrm{M}$ modified UTP and $200 \mu \mathrm{M}$ UTP. Incorporation rates for modified UTP ranged from $0.005 \mathrm{pmol} / \mathrm{ng}$ (for localization profile) to 0.044 $\mathrm{pmol} / \mathrm{ng}$ (for visualization of particles).

\section{Drosophila strains}

Ovaries from females expressing nls-GFP (Davis et al. 1995) were used to determine localization times, while ovaries from Oregon $\mathrm{R}$ females were used for particle tracking. Flies up to 24-h old were placed on yeast-supplemented food for 1-2 d prior to dissection in halocarbon 95 oil (Parton et al. 2010).

\section{Injection of RNA}

RNA was injected into oocytes as described by Van De Bor et al. (2005). Control RNA of known localization ability was injected into oocytes of each preparation to ensure that the properties attributed to different RNAs were not due to variation between oocytes. RNAs that were to be coinjected were mixed on ice at equimolar concentrations prior to injection, heated to $90^{\circ} \mathrm{C}$, returned to room temperature for $2 \mathrm{~min}$, and then stored on ice prior to injection. Colocalization was viewed in the Rhodamine and FITC channels without bleed-through.

\section{Microscopy}

Localization of injected RNA was analyzed by wide-field deconvolution microscopy using an Applied Precision Instruments Deltavision RT microscope. Images were captured with a CoolSnapHQ camera, processed using SoftWoRX, and deconvolved using 10 rounds of reiteration. A $\times 20$ (NA 0.75 ) dry objective was used to determine localization times and $\mathrm{a} \times 60$ (NA 1.4) water immersion objective for analysis of transport particles.

Colocalization of particles, particle size, and particle intensity measurements were made on the spinning disc (Yogawa CSU10) using a $\times 100$ (NA 1.45) oil objective. A beam splitter (Optisplit) allowed visualization of particles using the 488 and 561 channels simultaneously. Imaging (Andor iXon emccd camera) was tested for bleed-through. Acquisition speed was four to five frames per second (219 msec per frame); movies were assembled in ImagePro.

\section{Localization time}

RNAs labeled with Cy3-UTP were diluted to 120-150 ng/ $\mu$ L. Early to mid-stage 9 oocytes were dissected from nls-GFP flies, allowing visualization of the position of the oocyte nucleus prior to injection. Oocytes with a nucleus that was clearly visible were selected and focus was set prior to injection. A series of oocytes were injected, positioning the needle centrally. Injections near the oocyte nucleus were rejected. Movies where taken using the $\times 20$ objective at 1-min intervals for $50 \mathrm{~min}$ and analyzed visually for the time point beyond which the distribution of RNA at the anterior of the oocyte did not change in consecutive frames. We tried to determine localization times quantitatively by following the change in fluorescence intensity at the destination, but were unable to do so satisfactorily, as oocytes move and change shape during the time taken for an RNA to localize. The time taken to reach the pattern of localization beyond which little change occurred was taken as a measure of the overall kinetics of localization. While there was some natural variation between individual egg chambers in the proportion of dorso-ventral versus anterior localization, this was minor. The distribution of each localized RNA is shown in Supplemental Figure S3. Localization times were compared using Tukey's honest significance test.

\section{Particle tracking}

RNA at 120 or $250 \mathrm{ng} / \mu \mathrm{L}$ was injected into the center of stage 9 oocytes. Video micrographs were made at three to four frames per second. Movies were made starting shortly after injection and at time points from 15 to $20 \mathrm{~min}$ after injection to ensure that any variation in the behavior of particles with time was captured. Individual frames were converted to Tiff files, and the behavior of individual particles was analyzed using Metamorph image analysis software. A particle that moved was identified by eye and then its centroid was determined by the software, so that its movement between frames could be followed automatically. The position of the particle in subsequent frames was then exported to Microsoft Excel for import by ParticleStats software (Hamilton et al. 2010; http://www.particlestats.com/) to calculate the run length and average speed with which the particle had moved. Supplemental Figure S4 shows the movement of three particles containing IF1.7 RNA plotted by ParticleStats. At least 120 particles that moved for at least five consecutive frames were analyzed for each RNA. These included an even spread of particles observed from early to midway through localization and covered a similar range of oocytes stages and sizes in each case.

\section{SUPPLEMENTAL MATERIAL}

Supplemental material is available for this article. 


\section{ACKNOWLEDGMENTS}

This work was supported by a BBSRC project grant (F001045) awarded to D.J.F. and I.D., and a Wellcome Trust Senior Research Fellowship (067413) to I.D. We are grateful to David Kelley of the Wellcome Trust Centre for Cell Biology, University of Edinburgh, for help and advice in microscopy and data analysis; to Russell Hamilton, Department of Biochemistry, University of Oxford, for assistance with analysis of particle tracking data; to Suzanne McDermott for comments on the manuscript; and to reviewers who helped us to improve it.

Received February 11, 2011; accepted December 15, 2011.

\section{REFERENCES}

Becalska AN, Gavis ER. 2009. Lighting up mRNA localization in Drosophila oogenesis. Development 136: 2493-2503.

Besse F, Lopez de Quinto S, Marchand V, Trucco A, Ephrussi A. 2009. Drosophila PTB promotes formation of high-order RNP particles and represses oskar translation. Genes Dev 23: 195-207.

Bucheton A, Busseau I, Teninges D. 2002. I elements in Drosophila melanogaster. In Mobile DNA II (ed. NL Craig et al.), pp. 796-812. ASM Press, Washington, DC.

Bullock SL. 2007. Translocation of mRNAs by molecular motors: think complex? Semin Cell Dev Biol 18: 194-201.

Bullock SL, Ish-Horowicz D. 2001. Conserved signals and machinery for RNA transport in Drosophila oogenesis and embryogenesis. Nature 414: 611-616.

Bullock S, Bedau MA. 2006. Exploring the dynamics of adaptation with evolutionary activity plots. Artif Life 12: 193-197.

Bullock SL, Zicha D, Ish-Horowicz D. 2003. The Drosophila hairy RNA localization signal modulates the kinetics of cytoplasmic mRNA transport. EMBO J 22: 2484-2494.

Cech TR. 1990. Self-splicing of group I introns. Аnnu Rev Biochem 59: $543-568$.

Clark A, Meignin C, Davis I. 2007. A Dynein-dependent shortcut rapidly delivers axis determination transcripts into the Drosophila oocyte. Development 134: 1955-1965.

Cohen RS, Zhang S, Dollar GL. 2005. The positional, structural, and sequence requirements of the Drosophila TLS RNA localization element. RNA 11: 1017-1029.

Davis I, Ish-Horowicz D. 1991. Apical localization of pair-rule transcripts requires $3^{\prime}$ sequences and limits protein diffusion in the Drosophila blastoderm embryo. Cell 67: 927-940.

Davis I, Girdham CH, O'Farrell PH. 1995. A nuclear GFP that marks nuclei in living Drosophila embryos; maternal supply overcomes a delay in the appearance of zygotic fluorescence. Dev Biol 170: 726-729.

Delanoue R, Herpers B, Soetaert J, Davis I, Rabouille C. 2007. Drosophila Squid/hnRNP helps Dynein switch from a gurken mRNA transport motor to an ultrastructural static anchor in sponge bodies. Dev Cell 13: 523-538.

Dienstbier M, Boehl F, Li X, Bullock SL. 2009. Egalitarian is a selective RNA-binding protein linking mRNA localization signals to the dynein motor. Genes Dev 23: 1546-1558.

Ephrussi A, Dickinson LK, Lehmann R. 1991. Oskar organizes the germ plasm and directs localization of the posterior determinant nanos. Cell 66: 37-50.

Fawcett DH, Lister CK, Kellett E, Finnegan DJ. 1986. Transposable elements controlling I-R hybrid dysgenesis in D. melanogaster are similar to mammalian LINEs. Cell 47: 1007-1015.

Ferrandon D, Elphick L, Nusslein-Volhard C, St Johnston D. 1994. Staufen protein associates with the $3^{\prime} \mathrm{UTR}$ of bicoid mRNA to form particles that move in a microtubule-dependent manner. Cell 79: $1221-1232$
Ferrandon D, Koch I, Westhof E, Nusslein-Volhard C. 1997. RNARNA interaction is required for the formation of specific bicoid mRNA 3' UTR-STAUFEN ribonucleoprotein particles. EMBO J 16: $1751-1758$

Fusco D, Accornero N, Lavoie B, Shenoy SM, Blanchard JM, Singer RH, Bertrand E. 2003. Single mRNA molecules demonstrate probabilistic movement in living mammalian cells. Curr Biol 13: 161-167.

Hachet O, Ephrussi A. 2001. Drosophila Y14 shuttles to the posterior of the oocyte and is required for oskar mRNA transport. Curr Biol 11: $1666-1674$.

Hachet O, Ephrussi A. 2004. Splicing of oskar RNA in the nucleus is coupled to its cytoplasmic localization. Nature 428: 959-963.

Hamilton RS, Hartswood E, Vendra G, Jones C, Van De Bor V, Finnegan D, Davis I. 2009. A bioinformatics search pipeline, RNA2DSearch, identifies RNA localization elements in Drosophila retrotransposons. RNA 15: 200-207.

Hamilton RS, Parton RM, Oliveira RA, Vendra G, Ball G, Nasmyth K, Davis I. 2010. ParticleStats: open source software for the analysis of particle motility and cytoskeletal polarity. Nucleic Acids Res 38: W641-W646.

Hofacker IL, Priwitzer B, Stadler PF. 2004. Prediction of locally stable RNA secondary structures for genome-wide surveys. Bioinformatics 20: 186-190.

Jaeger L, Westhof E, Leontis NB. 2001. TectoRNA: modular assembly units for the construction of RNA nano-objects. Nucleic Acids Res 29: 455-463.

Jambor H, Brunel C, Ephrussi A. 2011. Dimerization of oskar 3' UTRs promotes hitchhiking for RNA localization in the Drosophila oocyte. RNA 17: 2049-2057.

Kampstra P. 2008. Beanplot: A boxplot alternative for visual comparison of distributions. J Stat Softw 28: 1-9.

Kim-Ha J, Smith JL, Macdonald PM. 1991. oskar mRNA is localized to the posterior pole of the Drosophila oocyte. Cell 66: 23-35.

Lan L, Lin S, Zhang S, Cohen RS. 2010. Evidence for a transport-trap mode of Drosophila melanogaster gurken mRNA localization. PLoS ONE 5: e15448. doi: 10.1371/journal.pone.0015448.

MacDougall N, Clark A, MacDougall E, Davis I. 2003. Drosophila gurken (TGF $\alpha$ ) mRNA localizes as particles that move within the oocyte in two dynein-dependent steps. Dev Cell 4: 307-319.

Mach JM, Lehmann R. 1997. An Egalitarian-BicaudalD complex is essential for oocyte specification and axis determination in Drosophila. Genes Dev 11: 423-435.

Najand N, Simmonds AJ. 2007. A minimal WLE2 element is not sufficient to direct apical localization in the absence of RNAs containing the full length wingless 3'UTR. RNA Biol 4: 138146.

Navarro C, Puthalakath H, Adams JM, Strasser A, Lehmann R. 2004. Egalitarian binds dynein light chain to establish oocyte polarity and maintain oocyte fate. Nat Cell Biol 6: 427-435.

Neuman-Silberberg FS, Schupbach T. 1993. The Drosophila dorsoventral patterning gene gurken produces a dorsally localized RNA and encodes a TGF $\alpha$-like protein. Cell 75: 165-174.

Parton RM, Valles AM, Dobbie IM, Davis I. 2010. Live cell imaging in Drosophila melanogaster. Cold Spring Harb Protoc doi: 10.1101/ pdb.top75.

Riechmann V, Ephrussi A. 2001. Axis formation during Drosophila oogenesis. Curr Opin Genet Dev 11: 374-383.

Rongo C, Gavis ER, Lehmann R. 1995. Localization of oskar RNA regulates oskar translation and requires Oskar protein. Development 121: 2737-2746.

Seleme MC, Disson O, Robin S, Brun C, Teninges D, Bucheton A. 2005. In vivo RNA localization of $I$ factor, a non-LTR retrotransposon, requires a cis-acting signal in ORF2 and ORF1 protein. Nucleic Acids Res 33: 776-785.

Spradling AC. 1993. Developmental genentics of oogenesis. In The development of Drosophila melanogaster, Vol. 1 (ed. M Bate, A 
Martinez Arias), pp. 1-70. Cold Spring Harbor Laboratory Press, Cold Spring Harbor, NY.

St Johnston D, Driever W, Berleth T, Richstein S, NussleinVolhard C. 1989. Multiple steps in the localization of bicoid RNA to the anterior pole of the Drosophila oocyte. Development 107: 13-19.

Tomancak P, Guichet A, Zavorszky P, Ephrussi A. 1998. Oocyte polarity depends on regulation of gurken by Vasa. Development 125: 1723-1732.

Van de Bor V, Davis I. 2004. mRNA localisation gets more complex. Curr Opin Cell Biol 16: 300-307.
Van De Bor V, Hartswood E, Jones C, Finnegan D, Davis I. 2005. gurken and the $I$ factor retrotransposon RNAs share common localization signals and machinery. Dev Cell 9: 51-62.

Wagner C, Palacios I, Jaeger L, St Johnston D, Ehresmann B, Ehresmann C, Brunel C. 2001. Dimerization of the 3'UTR of bicoid mRNA involves a two-step mechanism. J Mol Biol 313: 511-524.

Wilkie GS, Zimyanin V, Kirby R, Korey C, Francis-Lang H, Van Vactor D, Davis I. 2001. Small bristles, the Drosophila ortholog of NXF-1, is essential for mRNA export throughout development. RNA 7: 1781-1792. 

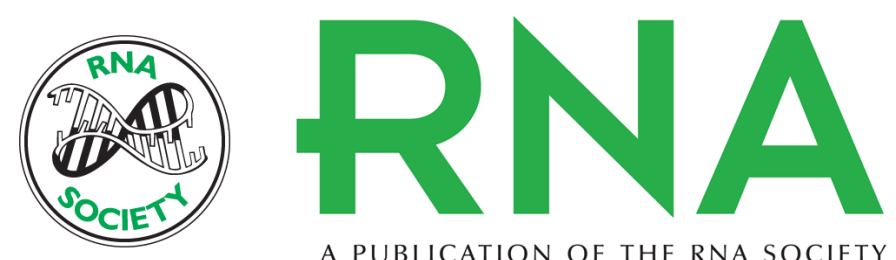

A PUBLICATION OF THE RNA SOCIETY

\section{RNA:RNA interaction can enhance RNA localization in Drosophila oocytes}

Eve Hartswood, Jim Brodie, Georgia Vendra, et al.

RNA 2012 18: 729-737 originally published online February 17, 2012

Access the most recent version at doi:10.1261/rna.026674.111

Supplemental http://rnajournal.cshlp.org/content/suppl/2012/02/02/rna.026674.111.DC1
Material

References This article cites 42 articles, 13 of which can be accessed free at: http://rnajournal.cshlp.org/content/18/4/729.full.html\#ref-list-1

\section{License}

Email Alerting Receive free email alerts when new articles cite this article - sign up in the box at the Service top right corner of the article or click here. 